\title{
On the Free Boson Gas in a Weak External Potential
}

\author{
M. Van den Berg ${ }^{1}$ and J. T. Lewis ${ }^{2}$ \\ 1 Institute for Theoretical Physics, State University of Groningen, 9700 AV Groningen, The \\ Netherlands \\ 2 Institute for Advanced Studies, Dublin, Ireland
}

\begin{abstract}
We calculate the equation of state and the barometric formula for a $d$-dimensional free boson gas in a weak external field of power form. We find that the condensate has a complicated structure in two dimensions.
\end{abstract}

\section{Introduction}

In this paper we investigate the phenomenon of condensation in a non-interacting boson gas in the presence of an external field, confined in a region of $d$-dimensional Euclidean space by a container with hard walls. It is well known that, in the absence of an external field, condensation cannot occur unless the dimension $d$ is greater than two. The result that an external field can induce condensation in one dimension was announced in [1]. Here we prove that if the external field comes from a scaled potential which is a positive power $\alpha$ of one coordinate, then the critical properties are determined by an effective dimension $d+2 / \alpha$; using this rule, the critical properties can be read-off from the list given for an ideal boson gas in arbitrary dimension by Ziff, Uhlenbeck and Kac [2].

The use of a scaled potential requires some explanation. It has been known for about fifty years that, in order to display a phase transition in a sharp way mathematically, it is necessary to pass to the thermodynamic limit. In the case of a free boson gas in a cubical container $C_{a}$ with side of length $a$, this is the limit in which $a$ increases without bound in comparison with the thermal wave-length $\lambda$ while the density remains fixed. On the other hand, since we are interested in the way in which the condensation phenomenon is modified by the presence of an external field, we must ensure that the effect of the potential $V(y)$ is not so great as to destroy the thermodynamic behaviour. This suggests that we should investigate the case in which $V(y)$ varies slowly as $y$ ranges over the container $C_{a}$; to express this mathematically, we write $V(y)$ as a function $\phi$ of $y / a$ and require that $\phi$ satisfies some local condition of slow variation. But the thermodynamic limit has to be taken, so the local condition on $\phi$ must be replaced by a global condition of slow variation. We expect results in the thermodynamic limit (under these 
conditions) to be a good approximation to those for a finite system in a container $C_{a}$ if both $\frac{a}{\lambda} \gg 1$ and $\beta v(a) \ll 1$, where $\beta$ is the inverse temperature and $v(a)$ is some measure of the variation in the potential over $C_{a}$.

In this paper we consider a potential $V(y)=c\left(\frac{y_{1}}{a}\right)^{\alpha}$ applied to a gas of noninteracting bosons, each of mass $m$, in a cube $[0, a]^{d}$ in $d$-dimensional Euclidean space. The single-particle hamiltonian $\widetilde{H}_{a}$ is given by

$$
\tilde{H}_{a}=-\frac{\hbar^{2}}{2 m} \sum_{i=1}^{d} \frac{\partial^{2}}{\partial y_{i}^{2}}+c\left(\frac{y_{1}}{a}\right)^{\alpha}, \quad \alpha>0,
$$

with Dirichlet boundary conditions on the cube $[0, a]^{d}$. We put $L=a\left(\hbar^{2} \beta / m\right)^{-1 / 2}$, $x_{i}=y_{i}\left(\hbar^{2} \beta / m\right)^{-1 / 2}$ and $\gamma=\beta c$; then $\beta \widetilde{H}_{a}$ is equivalent to

$$
H_{L}=-\frac{1}{2} \sum_{i=1}^{d} \frac{\partial^{2}}{\partial x_{i}^{2}}+\gamma\left(\frac{x_{1}}{L}\right)^{\alpha}
$$

with Dirichlet boundary conditions on $[0, L]^{d}$. Notice that two dimension-free parameters occur in (1.2): $L$ and $\gamma$. In this paper, the behaviour of the spectrum of $H_{L}$ as $L$ increases without bound is of crucial importance, but we do not discuss its dependence on $\gamma$. The order of the phase transition depends on $\alpha$ and $d$, but not on $\gamma$. The magnitudes of $\gamma$ and $L$ would become important if we were to estimate the error which would arise if the results obtained in the thermodynamic limit were applied to a finite system. We do not make this estimate here (except to show that for fixed $\gamma$ the error vanishes as $L$ increases without bound). In the mathematical sections of this paper we suppress the $\gamma$-dependences and put $\gamma=1$, and we work throughout with dimension-free variables as in (1.2). For the reader's convenience we state here the main results with the dimensions restored. Let $g_{r}(z)$ be the function defined on $[0,1)$ (and on $[0,1]$ for $r>1$ ) by

$$
g_{r}(z)=\sum_{n=1}^{\infty} \frac{z^{n}}{n^{r}}
$$

define a critical density $\rho_{c}$ by

$$
\lambda^{d} \rho_{c}=\left\{\begin{array}{l}
\int_{0}^{1} d u g_{d / 2}\left(e^{-\beta c u^{\alpha}}\right), \quad \frac{d}{2}+\frac{1}{\alpha}>1, \\
\infty, \text { otherwise. }
\end{array}\right.
$$

Here $\lambda$ is the thermal wave-length: $\lambda=\left(2 \pi \hbar^{2} \beta / m\right)^{1 / 2}$. When the mean density $\rho$ is greater than $\rho_{c}$, the grand canonical pressure $p$ is independent of $\rho$ and is given by

$$
\lambda^{d} \beta p=\int_{0}^{1} d u g_{d / 2+1}\left(e^{-\beta c u^{\alpha}}\right) ;
$$

when $\rho$ is less than $\rho_{c}$, p is determined parametrically as a function of $\rho$ by the pair 
of equations

$$
\left.\begin{array}{rl}
\lambda^{d} \beta p & =\int_{0}^{1} d u g_{d / 2+1}\left(\zeta e^{-\beta c u^{\alpha}}\right), \\
\lambda^{d} \rho & =\int_{0}^{1} d u g_{d / 2}\left(\zeta e^{-\beta c u^{\alpha}}\right) .
\end{array}\right\}
$$

Following standard rules, the thermodynamic functions are obtained from the expression for the pressure in terms of $\zeta$ and $\beta$ which is constructed from the corresponding one of the free gas, by use of the following rule: replace $g_{r}(\zeta)$ by 1

$\int_{0}^{1} \dot{d} u g_{r}\left(\zeta e^{-\beta c u^{\alpha}}\right)$; we may regard this as an averaging over a local chemical potential.

There are two main strategies for the rigorous investigation of boson condensation: the first is to estimate the uncondensed fraction and to derive the equation of state. The second is to estimate the condensed fraction by investigating the asymptotic behaviour of the fractional occupation of the low-lying levels as the thermodynamic limit is approached; this is technically more difficult, but gives more information (for instance, a proof of the barometric formula). The first strategy was used by Davies [3] and by Landau and Wilde [4]. The second was outlined by $\mathrm{Kac}$ in unpublished lecture notes, worked out in detail in a variety of cases in [5-7], [8] and eventually published in [2]. In this paper we adopt the second strategy; we are thus able to investigate the complicated structure of the condensate which is possible in the two-dimensional case in the presence of an external field. Above the critical density there is macroscopic occupation of the ground-state in dimensions one, three, four, ... ; in dimension two there is macroscopic occupation of an infinite number of low-lying levels. This is discussed in detail in Sect. 2. In each of Sects. 2, 3 and 4, we state a mathematical result, discuss its physical consequences and give a sketch of the proof. The details of the proofs are reserved for Sect. 6.

\section{The Occupation Numbers}

Let $\varepsilon_{1}^{L}<\varepsilon_{2}^{L}<\ldots$ be the eigenvalues of

$$
h_{L}=-\frac{1}{2} \frac{d^{2}}{d x_{1}^{2}}+\left(\frac{x_{1}}{L}\right)^{\alpha}
$$

with Dirichlet boundary conditions on $[0, L]$, where $\alpha$ is a positive real number; Let $\Phi_{1}^{L}, \Phi_{2}^{L}, \ldots$ be the corresponding normalized eigenfunctions. Denote by $k$ the multi-index $\left(k_{1}, \ldots, k_{d}\right)$, and let $\left\{E_{k}^{L}\right\}$ be the eigenvalues of the single-particle hamiltonian

$$
H_{L}=-\frac{1}{2} \Delta+\left(\frac{x_{1}}{L}\right)^{\alpha}
$$


with Dirichlet boundary conditions on the cube

$$
\begin{gathered}
B_{L}=\left\{x \in \mathbb{R}^{d}: 0 \leqq x_{1} \leqq L, \ldots, 0 \leqq x_{d} \leqq L\right\} ; \text { then } \\
E_{k}^{L}=\varepsilon_{k_{1}}^{L}+\frac{\pi^{2}}{2 L^{2}} \sum_{m=2}^{d} k_{m}^{2},
\end{gathered}
$$

and the corresponding eigenfunction $\Psi_{k}^{L}$ is given by

$$
\Psi_{k}^{L}(x)=\Phi_{k_{1}}^{L}\left(x_{1}\right) \prod_{m=2}^{d}\left(\frac{2}{L}\right)^{1 / 2} \sin \left(\pi k_{m} x_{m} / L\right) .
$$

In the grand canonical ensemble for non-interacting bosons in the cube $B_{L}$, the mean occupation number $\left\langle n_{k}\right\rangle_{L}$ of the single-particle level with multi-index $k$ is given by (see [2], for example)

$$
\left\langle n_{k}\right\rangle_{L}=\zeta(L)\left(e^{\eta_{k}^{L}}-\zeta(L)\right)^{-1}
$$

where

$$
\eta_{k}^{L}=E_{k}^{L}-E_{1}^{L}
$$

$E_{1}^{L}$ denotes the eigenvalue with multi-index $(1,1, \ldots, 1)$, and $\zeta(L)$ is determined by the condition that the mean number $\langle N\rangle_{L}$ of bosons is given by

$$
\sum_{k}\left\langle n_{k}\right\rangle_{L}=\langle N\rangle_{L}
$$

The thermodynamic limit consists in letting $L$ increase without bound while the mean density $\rho=L^{-d}\langle N\rangle_{L}$ is kept fixed. Let $\rho_{c}$ be the critical density defined by

$$
\rho_{c}=\left\{\begin{array}{cl}
(2 \pi)^{-d / 2} \int_{0}^{1} d u g_{d / 2}\left(e^{-u^{\alpha}}\right), & \frac{d}{2}+\frac{1}{\alpha}>1 \\
\infty & , \text { otherwise }
\end{array}\right.
$$

The following result is crucial:

Theorem 1. The limit $\zeta=\lim _{L \rightarrow \infty} \zeta(L)$ exists for all values of $\rho$; for $\rho<\rho_{c}$ it is the unique root of the equation

$$
\int_{0}^{1} d u g_{d / 2}\left(\zeta e^{-u^{\alpha}}\right)=(2 \pi)^{d / 2} \rho
$$

while for $\rho \geqq \rho_{c}$ we have $\zeta=1$.

Furthermore, for $\rho>\rho_{c}$ we have

$$
\zeta(L) \sim \begin{cases}1-\frac{1}{\left(\rho-\rho_{c}\right) L^{d}}, & d=1,3,4, \ldots \\ 1-\frac{1}{A L^{2}} \quad, \quad d=2,\end{cases}
$$


where $A$ is the unique positive root of the equation

$$
\sum_{j=1}^{\infty}\left\{\frac{\left(j^{2}-1\right) \pi^{2}}{2}+\frac{1}{A}\right\}^{-1}=\rho-\rho_{c} .
$$

At this point we note the following immediate consequence: above the critical density there is macroscopic occupation of the ground state in case $d=1,3,4, \ldots$ while for $d=2$ there is macroscopic occupation of an infinite number of low-lying levels. In detail, we have (for $\rho>\rho_{c}$ )

$$
\begin{aligned}
& \lim _{L \rightarrow \infty} L^{-d}\left\langle n_{1}\right\rangle_{L}=\left(\rho-\rho_{c}\right), \quad d=1,3,4, \ldots \\
& \lim _{L \rightarrow \infty} L^{-2}\left\langle n_{1, j}\right\rangle_{L}=\left\{\left(j^{2}-1\right) \frac{\pi^{2}}{2}+\frac{1}{A}\right\}^{-1}, \quad d=2,
\end{aligned}
$$

where $A$ is the unique positive root of Eq. (2.11). The density of each of the other levels tends to zero. For $\rho$ less than $\rho_{c}$ we have $\zeta(L)$ strictly less than one, so that

$$
\lim _{L \rightarrow \infty} \mathrm{L}^{-d}\left\langle n_{k}\right\rangle_{L}=0, \quad d=1,2,3, \ldots,
$$

for every multi-index $k$.

The proof of Theorem 1 is given in Sect. 6; we sketch the idea here. We expand $\rho=\langle N\rangle_{L} L^{-d}$ as a power-series in $\zeta(L)$; by expanding each $\left\langle n_{k}\right\rangle_{L}$ with $k_{1} \neq 1$ (we isolate the $\left\langle n_{k}\right\rangle_{L}$ with $k_{1}=1$ because they may become singular as $\zeta(L)$ approaches one)

$$
\rho=\frac{1}{L^{d}} \sum_{\left\{k: k_{1}=1\right\}}\left\langle n_{k}\right\rangle_{L}+\sum_{n=1}^{\infty} \zeta(L)^{n} S_{L}^{\prime}(n),
$$

where

$$
S_{L}^{\prime}(t)=\frac{1}{L^{d}} \sum_{\left\{k: k_{1} \neq 1\right\}} e^{-t \eta_{k}^{L}} .
$$

The asymptotic properties of $S_{1}^{\prime}(t)$ for small $t$ depend on those of $\eta_{k}^{1}$ for large $|k|$ and can be got from the Feyman-Kac formula (see [11]). If we are careful, we can trade-off the decrease of $\eta_{k}^{L}$ with $L$ against the increase of $n$ to prove that, for $\rho<\rho_{c}$,

$$
\lim _{L \rightarrow \infty} \sum_{n=1}^{\infty} \zeta(L)^{n} S_{L}^{\prime}(n)=(2 \pi)^{-d / 2} \int_{0}^{1} d u g_{d / 2}\left(\zeta e^{-u^{\alpha}}\right),
$$

where $\zeta$ is the root of Eq. (2.9). Hence $L^{-d} \sum_{\left\{k: k_{1}=1\right\}}\left\langle n_{k}\right\rangle_{L}$ tends to zero (and so does $L^{-d}\left\langle n_{k}\right\rangle_{L}$ for arbitrary $k$, since the convergence of the series implies that $S_{L}^{\prime}(n)$ tends to zero). Similarly, we can show that, for $\rho>\rho_{c}, \lim _{L \rightarrow \infty} \sum_{n=1}^{\infty} \zeta(L)^{n} S_{L}^{\prime}(n)=\rho_{c}$ so that $L^{-d} \sum_{\left\{k: k_{1}=1\right\}}\left\langle n_{k}\right\rangle_{L}$ tends to $\rho-\rho_{c}$ and $\zeta(L)$ tends to one. The asymptotic behaviour of the occupation numbers follows from the explicit dependence of $\eta_{k}^{L}$ on $L$; in Lemma 1 of Sect. 6 we prove that, for large $L$,

$$
\varepsilon_{j}^{L} \sim L^{-2 \alpha(\alpha+2)} A_{j}
$$


where $A_{j}$ is the $j$ th eigenvalue of

$$
h=-\frac{1}{2} \frac{d^{2}}{d u^{2}}+u^{\alpha}
$$

with Dirichlet boundary conditions on $[0, \infty)$. Hence

$$
\eta_{k}^{L} \sim L^{-2 \alpha /(\alpha+2)}\left(A_{k_{1}}-A_{1}\right)+\frac{\pi^{2}}{2 L^{2}} \sum_{m=2}^{d}\left(k_{m}^{2}-1\right) .
$$

\section{The Equation of State}

In the grand canonical ensemble for non-interacting bosons in the cube $B_{L}$, the pressure $p_{L}$ is given by (see [2], for example)

$$
p_{L}=-L^{-d} \sum_{k} \log \left(1-\zeta(L) e^{-\eta_{k}^{L}}\right),
$$

where $\zeta(L)$ is determined by condition (2.7). We have the following result:

Theorem 2. The limit $p=\lim _{L \rightarrow \infty} p_{L}$ exists and is given $b y$

$$
p=(2 \pi)^{-d / 2} \int_{0}^{1} d u g_{d / 2+1}\left(\zeta e^{-u^{\alpha}}\right),
$$

where $\zeta$ is given in the statement of Theorem 1 .

From this we can deduce the properties of the pressure $p$ as a function of the density $\rho$. Above the critical density $\rho_{c}$, the pressure is independent of the density and is given by

$$
p=(2 \pi)^{-d / 2} \int_{0}^{1} d u g_{d / 2+1}\left(e^{-u^{\alpha}}\right) .
$$

Below the critical density it is determined parametrically by the pair of equations

$$
\left.\begin{array}{l}
p=(2 \pi)^{-d / 2} \int_{0}^{1} d u g_{d / 2+1}\left(\zeta e^{-u^{\alpha}}\right), \\
\rho=(2 \pi)^{-d / 2} \int_{0}^{1} d u g_{d / 2}\left(\zeta e^{-u^{\alpha}}\right) .
\end{array}\right\}
$$

Since the critical behaviour of the thermodynamic functions is determined by the asymptotic properties for large $n$ of the coefficient of $\zeta^{n}$ in their power-series expansion, it is readily seen that the critical behaviour in dimension $d$ in the presence of a potential $\left(\frac{x_{1}}{L}\right)^{\alpha}$ is the same as the critical behaviour of the free boson gas in dimension $d+\frac{2}{\alpha}$. Using this effective dimension, we may read-off 
the critical behaviour of the thermodynamic functions in the presence of an external field from the list in [2].

\section{The Barometric Formula}

In this section we investigate the spatial distribution of the boson gas in the thermodynamic limit. For reasons which should become clear in the discussion, we introduce a scaled local density $v_{1}^{L}(u)$ defined, for $u$ in $[0,1]^{d}$, by

$$
v_{1}^{L}(u)=\langle N(L u)\rangle_{L}=\sum_{k} \frac{\zeta(L)}{e^{\eta_{k}^{L}}-\zeta(L)}\left(\Psi_{k}^{L}(L u)\right)^{2} .
$$

Theorem 3. The limit $v_{1}(u)=\operatorname{Lim}_{L \rightarrow \infty} v_{1}^{L}(u)$ exists (in the sense of the weak convergence of distribution functions) and is given, for $\rho<\rho_{c}$, by

$$
v_{1}(u)=(2 \pi)^{-d / 2} g_{d / 2}\left(\zeta e^{-u_{1}^{\alpha}}\right), \quad d=1,2,3, \ldots
$$

For $\rho \geqq \rho_{c}$ we have:

$$
\begin{gathered}
d=1: v_{1}(u)=\left(\rho-\rho_{c}\right) \delta\left(u_{1}\right)+(2 \pi)^{-1 / 2} g_{1 / 2}\left(e^{-u_{1}^{\alpha}}\right) \\
d=2: v_{1}(u)=\delta\left(u_{1}\right)\left\{\sum_{j=1}^{\infty} \frac{2 \sin ^{2} \pi j u_{2}}{\left.\left(j^{2}-1\right) \frac{\pi^{2}}{2}+\frac{1}{A}\right\}+(2 \pi)^{-1} g_{1}\left(e^{-u_{1}^{\alpha}}\right)}\right. \\
d \geqq 3: v_{1}(u)=\left(\rho-\rho_{c}\right) \delta\left(u_{1}\right) 2^{d-1} \prod_{m=2}^{d} \sin ^{2} \pi u_{m}+(2 \pi)^{-d / 2} g_{d / 2}\left(e^{-u_{1}^{\alpha}}\right) .
\end{gathered}
$$

Here $\delta\left(u_{1}\right)$ is the Dirac measure supported on the hyperplane $u_{1}=0$.

In a similar fashion, a scaled hydrostatic pressure $p^{L}(u)$ can be defined by

$$
p^{L}(u)=-\sum_{k} \log \left(1-\zeta(L) e^{-\eta_{k}^{L}}\right)\left(\Psi_{k}^{L}(L u)\right)^{2}
$$

for $u$ in $[0,1]^{d}$. We state without proof

Theorem 4. The limit $p(u)=\lim p^{L}(u)$ exists for each $u$ in $[0,1]^{d}$ and is given by $L \rightarrow \infty$

$$
p(u)=(2 \pi)^{-d / 2} g_{d / 2+1}\left(\zeta e^{-u_{1}^{\alpha}}\right) .
$$

We note that the mean density $\rho$ is the average of the scaled local density $v_{1}(u)$ :

$$
\rho=\int_{[0,1]^{d}} v_{1}(u) d u
$$

and the grand canonical pressure $p$ is the average of the hydrostatic pressure $p(u)$ :

$$
p=\int_{[0,1]^{d}} p(u) d u \text {. }
$$


To understand the choice of scaling in the definition of $v_{1}$, it is useful to note the following additional results (which we state without proof): For fixed $x$ in $[0, L]^{d}$ the unscaled local density coincides, in the thermodynamic limit, with that for the free boson gas:

$$
\lim _{L \rightarrow \infty}\langle N(x)\rangle_{L}=\lim _{L \rightarrow \infty}\langle N(x)\rangle_{L}^{0},
$$

where \langle\rangle$_{L}^{0}$ denotes the grand canonical average in the absence of an external potential. For $x$ fixed, the potential $\left(\frac{x}{L}\right)^{\alpha}$ becomes small as $x$ increases and its effect on the distribution disappears. To see the barometric distribution of the normal fluid we have to look at the density at a point which moves out as $L$ increases. We do this when we compute $v_{1}(u)$. However, on this scale the condensate is concentrated on the hyperplane $u_{1}=0$ and described there by a global wavefunction.

If we are interested in the spatial distribution of the condensate we have to choose another scaling: define $v_{2}^{L}(u)$ for $u=\left(u_{1}, \ldots, u_{d}\right)$ in $\left[0, L^{2 /(\alpha+2)}\right] \times[0,1]^{d-1}$ by $v_{2}^{L}(u)=L^{-2 /(\alpha+2)}\left\langle N\left(L^{\alpha /(\alpha+2)} u_{1}, L u_{2}, \ldots, L u_{d}\right)\right\rangle_{L} ;$ then $v_{2}(u)=\lim _{L \rightarrow \infty} v_{2}^{L}(u)$ exists and is given, for $\rho \geqq \rho_{\mathrm{c}}$, by

$$
v_{2}(u)=\left\{\begin{array}{cl}
\left(\rho-\rho_{c}\right) \chi_{1}^{2}\left(u_{1}\right) 2^{d-1} \prod_{m=2}^{d} \sin ^{2} \pi u_{m}, & d=1,3,4, \ldots \\
\chi_{1}^{2}\left(u_{1}\right)\left\{\sum_{j=1}^{\infty} \frac{2 \sin ^{2} \pi u_{2} j}{\left.\left(j^{2}-1\right) \frac{\pi^{2}}{2}+\frac{1}{A}\right\},}\right. & d=2,
\end{array}\right.
$$

where $\chi_{1}$ is the ground-state wave-function of $-\frac{1}{2} \frac{d^{2}}{d u_{1}^{2}}+u_{1}^{\alpha}$ with Dirichlet boundary conditions on $[0, \infty]$. For $\rho<\rho_{c}$, we have $v_{2}(u) \equiv 0$. In this scaling the normal fluid has disappeared while the condensate distribution is described by macroscopic wave-functions.

The results stated in this section are proved using a modification of the technique described in Sect. 3. We use the Feynman-Kac formula to get the asymptotic properties of

$$
\frac{1}{L^{d}} \sum_{\left\{k: k_{1} \neq 1\right\}} e^{-t \eta_{k}^{L}}\left(\Psi_{k}^{L}(x)\right)^{2}
$$

and make use of the scaling properties of the eigenfunctions $\Psi_{k}^{L}$ as well as those of the eigenvalues.

\section{The Kac Density}

In order to discuss the fluctuations in those levels which become macroscopically 
occupied, it is convenient to calculate

$$
\left\langle e^{-z N / L^{d}}\right\rangle_{L}
$$

in the thermodynamic limit. The result is given in

Theorem 5. The following limit exists

$$
\lim _{L \rightarrow \infty}\left\langle e^{-z N / L^{d}}\right\rangle_{L}=\left\{\begin{array}{lc}
e^{-z \rho}, & \rho<\rho_{c}, \quad d=1,2,3, \ldots \\
\frac{e^{-z \rho_{c}}}{1+z\left(\rho-\rho_{c}\right)} & \rho>\rho_{c}, \quad d=1,3, \ldots \\
\frac{e^{-z \rho_{c}}\left(2 z-\pi^{2}+2 / A\right)^{1 / 2}}{\left(2 / A-\pi^{2}\right)^{1 / 2}} \frac{\sinh \left(2 / A-\pi^{2}\right)^{1 / 2}}{\sinh \left(2 z-\pi^{2}+2 / A\right)^{1 / 2}}, & \rho>\rho_{c}, \quad d=2,
\end{array}\right.
$$

where $A$ and $\rho_{c}$ are as in Theorem 1.

We will not prove Theorem 5 in this paper since the proof is along the same lines as in [2]; however, a discussion of its consequences is justified in the twodimensional case, in which a set of low-lying levels becomes macroscopically occupied. If we expand both sides of (5.1) in powers of $z$ we obtain for $\rho>\rho_{c}$

$$
\begin{array}{ll}
\lim _{L \rightarrow \infty}\left\langle\left(\frac{N}{L^{d}}-\rho_{c}\right)^{2}\right\rangle_{L}=2\left(\rho-\rho_{c}\right)^{2}, & d=1,3, \ldots \\
\lim _{L \rightarrow \infty}\left\langle\left(\frac{N}{L^{2}}-\rho_{c}\right)^{2}\right\rangle_{L}=2\left(\rho-\rho_{c}\right)^{2}+\frac{3\left(\rho-\rho_{c}-1 / 3\right)}{2 / A-\pi^{2}}, & d=2 .
\end{array}
$$

So we have in particular for $d=2$

$$
\lim _{L \rightarrow \infty}\left\langle\left(\frac{N}{L^{2}}-\rho_{c}\right)^{2}\right\rangle_{L} \sim \begin{cases}\left(\rho-\rho_{c}\right)^{2}, & \rho \gtrsim \rho_{c} \\ 2\left(\rho-\rho_{c}\right)^{2}, & \rho \gg \rho_{c} .\end{cases}
$$

The behaviour of $\lim _{L \rightarrow \infty}\left\langle\left(\frac{N}{L^{2}}-\rho_{c}\right)^{2}\right\rangle_{L}$ for $d=2$ can be qualitatively understood as follows: For $\rho$ much larger than $\rho_{c}$ it follows from the estimates in Theorem 1 that $\left.\left\langle n_{1,1}\right\rangle_{L}\right\rangle\left\langle n_{1, j}\right\rangle_{L}$ for $j=2,3, \ldots$ and $L$ large, so the main fluctuation occurs in one energy level; therefore it should behave like the cases $d=1,3, \ldots$. But for $\rho-\rho_{c}$ small and positive $\left\langle n_{1,1}\right\rangle$ is of the same order as $\left\langle n_{1, j}\right\rangle, j=2, \ldots$, so the total fluctuation is reduced. The Kac density $v(x, \rho)$ is related to $\lim \left\langle e^{-z N / L^{d}}\right\rangle_{L}$ as follows $L \rightarrow \infty$

$$
\lim _{L \rightarrow \infty}\left\langle e^{-z N / L^{d}}\right\rangle_{L}=\int_{0}^{\infty} v(x, \rho) e^{-z x} d x
$$


Inverting (5.4) we obtain:

$$
\begin{gathered}
\rho<\rho_{c}: v(x, \rho)=\delta(x-\rho) ; \rho>\rho_{c}, x<\rho_{c}: v(x, \rho)=0 \\
\rho>\rho_{c}, x>\rho_{c}, \quad d=1,3,4, \ldots: v(x, \rho)=\frac{1}{\rho-\rho_{c}} \quad e^{-\left(x-\rho_{c}\right) /\left(\rho-\rho_{c}\right)} \\
\rho>\rho_{c}, x>\rho_{c}, \quad d=2: \\
v(x, \rho)=\frac{\pi^{2} \sinh \left(2 / A-\pi^{2}\right)^{1 / 2}}{\left(2 / A-\pi^{2}\right)^{1 / 2}} \sum_{n=1}^{\infty}(-1)^{n-1} n^{2} e^{-\left(x-\rho_{c}\right) \cdot\left(\pi^{2} / 2\right)\left(n^{2}+\left(2 / A \pi^{2}\right)-1\right)}
\end{gathered}
$$

In principle, the expression (5.5) can be used to decompose any grand canonical average $\lim \langle F\rangle_{L}$ into the corresponding canonical average at a fixed density $x$. $L \rightarrow \infty$

\section{The Promised Proofs}

In this section we give the promised proofs. To do so we require estimates on the lowest two eigenvalues of $h_{L}$ : these will be presented in Lemma 1. In Lemma 2 we recall some estimates on the asymptotic behaviour of the eigenvalues and eigenfunctions of the one-particle hamiltonian. We will not prove this lemma since the proofs can be found elsewhere [9]. Lemma 3 is a consequence of Lemmas 1 and 2. To prove Lemma 4 we will use again the estimates on the lowest two eigenvalues (Lemma 1) and Lemma 3. Theorem 1 follows from Lemma 4 after a few steps.

Lemma 1. There exist three positive constants $a_{1}, a_{2}$ and $a_{3}$ such that

$$
\left.\begin{array}{l}
\varepsilon_{1}^{L} \leqq a_{1} L^{-2 \alpha /(\alpha+2)} \\
a_{2} L^{-2 \alpha /(\alpha+2)} \leqq \varepsilon_{2}^{L}-\varepsilon_{1}^{L} \leqq a_{3} L^{-2 \alpha /(\alpha+2)}
\end{array}\right\}
$$

where $\varepsilon_{1}^{L}<\varepsilon_{2}^{L}<\ldots$ are the eigenvalues of $h_{L}=-\frac{1}{2} \frac{d^{2}}{d x^{2}}+\left(\frac{x}{L}\right)^{\alpha}$ on $[0, L]$ with Dirichlet boundary conditions on $[0, L]$.

Proof of Lemma 1 . We substitute $x=L^{\alpha /(\alpha+2)} y$ in the eigenvalue equation $h_{L} \Phi_{j}^{L}(x)=\varepsilon_{j}^{L} \Phi_{j}^{L}(x)$. So we obtain the equation

$$
-\frac{1}{2} \frac{d^{2} \tilde{\Phi}_{j}^{L}}{d y^{2}}+y^{\alpha} \tilde{\Phi}_{j}^{L}=A_{j}^{L} \tilde{\Phi}_{j}^{L}
$$

with Dirichlet boundary conditions on $\left[0, L^{2 /(\alpha+2)}\right]$ and with $A_{j}^{L}=L^{2 \alpha /(\alpha+2)} \varepsilon_{j}^{L}$, so $A_{j}^{L}<A_{j}^{L^{\prime}}$ if $L^{\prime}<L$. So in particular we choose $a_{1}=A_{1}^{1}$ for $L>L^{\prime}=1$ and $a_{3}=A_{1}^{1}+A_{2}^{1}$ for $L>1$. Furthermore for $L$ large enough we may choose $a_{2}=\frac{1}{2}$ $\left(A_{2}^{\infty}-A_{1}^{\infty}\right)$.

Lemma 2. Let $V(x)$ be a non-negative Borel measurable function defined on a finite region $B \subset R^{d}$ and let $V(x)$ satisfy a Lipschitz condition of the form $\left|V(x)-V\left(x^{\prime}\right)\right|<M\left|x-x^{\prime}\right|^{\alpha}$ for some $0<\alpha \leqq 1$ and for almost all pairs $x \subset B$ and $x^{\prime} \subset B$, and let $\left\{E_{j}\right\}$ denote the spectrum of $-\frac{\Delta}{2}+V(x)$ with a corresponding 
orthonormal set of eigenfunctions $\left\{\Phi_{j}\right\}$ with Dirichlet conditions on the boundary $\partial B$ of $B$, then if the boundary $\partial B$ of $B$ is regular we have for all $t>0$ the following:

$$
\begin{aligned}
& \sum_{j=1}^{\infty} e^{-t E_{j}} \leqq \frac{1}{(2 \pi t)^{d / 2}} \int_{x \in B} e^{-t V(x)} d x, \\
& \left|\sum_{j=1}^{\infty} e^{-t E_{j}}-\frac{1}{(2 \pi t)^{d / 2}} \int_{x \in B} e^{-t V(x)} d x\right| \leqq \frac{1}{(2 \pi t)^{d / 2}} \int_{x \in B} d x e^{-t V(x)} \\
& \left\{2 d e^{-2 d^{2} / d t}+\left|\int_{0}^{t} d \tau \int_{u \in B} d u(V(u)-V(x)) e^{-t|x-u|^{2} / 2 \tau(t-\tau)} \cdot\left(\frac{t}{2 \pi \tau(t-\tau)}\right)^{d / 2}\right|\right\}, \\
& \sum_{j=1}^{\infty} e^{-t E_{j}} \Phi_{j}^{2}(x) \leqq \frac{1}{(2 \pi t)^{d / 2}} \int_{0}^{t} \frac{d \tau}{t} \int_{u \in B} d u \cdot e^{-t V(u)-t|x-u|^{2} / 2 \pi(t-\tau)}\left(\frac{t}{2 \pi \tau(t-\tau)}\right)^{d / 2}, \\
& \left|\sum_{j=1}^{\infty} e^{-t E_{j}} \Phi_{j}^{2}(x)-\frac{e^{-t V(x)}}{(2 \pi t)^{d / 2}}\right| \\
& \leqq \frac{e^{-t V(x)}}{(2 \pi t)^{d / 2}}\left\{2 d e^{-2 d_{x}^{2} / d t}+\left|\int_{0}^{t} d \tau \int_{u \in B} d u(V(u)-V(x)) \cdot\left(\frac{t}{2 \pi \tau(t-\tau)}\right)^{d / 2} e^{-t|x-u|^{2} / 2 \tau(t-\tau)}\right|\right. \\
& \left.+\left|\int_{0}^{t} \frac{d \tau}{t} \int_{u \in B} d u\left(\frac{t}{2 \pi \tau(t-\tau)}\right)^{d / 2} e^{t(V(x)-V(u))-t|x-u|^{2} / 2 \tau(t-\tau)}-1\right|\right\},
\end{aligned}
$$

where $d_{x}=\inf _{y \in \partial B}|y-x|$.

Before Lemma 3 we give some definitions:

Definitions. Let

$$
\begin{aligned}
& S_{L}(t)=\frac{1}{L^{d}} \sum_{k} e^{-t \eta_{k}^{L}} \\
& S_{L}^{\prime}(t)=\frac{1}{L^{d}} \sum_{\left\{k: k_{1} \neq 1\right\}} e^{-t \eta_{k}^{L}} \\
& G(t)=\frac{1}{(2 \pi t)^{d / 2}} \int_{0}^{1} d u e^{-t u^{\alpha}} \\
& f_{L}(z)=\sum_{n=1}^{\infty} z^{n} S_{L}^{\prime}(n)
\end{aligned}
$$

and let $T(L)$ be an integer such that for some $r_{1}>1$

$$
T(L) \leqq \frac{r_{1}}{a_{2}} L^{2 \alpha /(\alpha+2)} \leqq T(L)+1 .
$$

Lemma 3. There exists a set of constants $r_{2}>1, r_{3}>0, r_{4}>1$ etc. such that for all $t<T(L)$

$$
\left|S_{L}(t)-G(t)\right| \leqq \frac{a_{4}}{t^{r_{2}} L^{r_{3}}}+\frac{a_{5}}{t^{r_{4}} L^{r_{5}}}+\ldots \frac{a_{8}}{t^{r_{10}} L^{r_{11}}},
$$

where $a_{4}, a_{5}, \ldots$ are finite positive constants. 
Proof. We have the following estimate:

$$
\begin{aligned}
\mid S_{L}(t) & -G(t)|=| e^{t E_{1}^{L}} \cdot \frac{1}{L^{d}} \sum_{\{k\}} e^{-t E_{k}^{L}}-G(t) \mid \\
& \leqq \frac{1}{L^{d}}\left(e^{t E_{1}^{L}}-1\right) \sum_{\{k\}} e^{-t E_{k}^{L}}+\left|\frac{1}{L^{d}} \sum_{\{k\}} e^{-t E_{k}^{L}}-G(t)\right| .
\end{aligned}
$$

Moreover by the inequality $e^{x}-1 \leqq x e^{x}$ and by (6.3) and by (6.1) we have

$$
\begin{aligned}
& \frac{1}{L^{d}}\left(e^{t E_{1}^{L}}-1\right) \cdot \sum_{\{k\}} e^{-t E_{k}^{L}} \\
& \quad \leqq e^{r_{1} a_{1} / a_{2}+(d-1) r_{1} \pi^{2} / 2 a_{2}} t^{1-d / 2-1 / \alpha}\left(\frac{a_{1}}{L^{2 \alpha /(\alpha+2)}}+\frac{(d-1) \pi^{2}}{2 L^{2}}\right) \Gamma\left(1+\frac{1}{\alpha}\right)
\end{aligned}
$$

$G(t) \leqq \Gamma\left(1+\frac{1}{\alpha}\right) t^{-d / 2-1 / \alpha}$. We require $r_{2}+1-d / 2-1 / \alpha>0$ in order to write

$$
\frac{t^{1-d / 2-1 / \alpha}}{L^{2 \alpha /(\alpha+2)}}=\frac{t^{1-d / 2-1 / \alpha+r_{2}}}{t^{r_{2}} L^{2 \alpha /(\alpha+2)}} \leqq \frac{a_{4}}{t^{r_{2}}} \cdot L^{\left(-d / 2-1 / \alpha+r_{2}\right) 2 \alpha /(\alpha+2)} .
$$

Furthermore, in order to satisfy the lemma we require also $r_{2}>1$ and $-d / 2-$ $1 / \alpha+r_{2}<0$. These three requirements can be satisfied for some $r_{2}$ if $d / 2+1 / \alpha>1$. To estimate the remaining term we use (6.4) so

$$
\begin{aligned}
& \left|\frac{1}{L^{d}} \sum_{\{k\}} e^{-t E_{k}^{L}}-G(t)\right| \\
& \leqq \frac{1}{(2 \pi t)^{d / 2}} \int_{u \in B_{1}} d u e^{-t u_{1}^{\alpha}}\left\{2 d e^{-2 L^{2} d^{2} u, 1 / d t}\right. \\
& \left.+\int_{v \in B_{1}} d v \int_{0}^{t} d \tau e^{-t L^{2}|v-u|^{2} / 2 \tau(t-\tau)}\left|v_{1}^{\alpha}-u_{1}^{\alpha}\right| \cdot\left(\frac{t L^{2}}{2 \pi \tau(t-\tau)}\right)^{d / 2}\right\} \\
& \leqq \frac{1}{(2 \pi t)^{d / 2}} \int_{u \in B_{1}} d u e^{-t u_{1}^{\alpha}}\left\{2 d e^{-2 L^{2} d_{u, 1}^{2} / d t}\right. \\
& \left.+\int_{0}^{1} d v_{1} \int_{0}^{t} d \tau e^{-t L^{2}\left|v_{1}-u_{1}\right|^{2} / 2 \tau(t-\tau)}\left(\frac{t L^{2}}{2 \pi \tau(t-\tau)}\right)^{1 / 2}\right\},
\end{aligned}
$$

where $B_{1}=\left\{u: 0 \leqq u_{1} \leqq 1, \ldots, 0 \leqq u_{d} \leqq 1\right\}$ and $d_{u, 1}=\inf _{w \in \partial B_{1}}|u-w|$. Since

$$
\begin{gathered}
\frac{2 d}{(2 \pi t)^{d / 2}} \int_{u \in B_{1}} d u e^{-t u_{1}^{\alpha}-2 L^{2} d_{u, 1}^{2} / d t}{ }^{\leqq} \frac{2 d}{(2 \pi t)^{d / 2}}\left\{\int_{d_{u, 1}>L^{-1 / 2}} d u e^{-t u_{1}^{\alpha}-2 L^{2}-d_{u, 1}^{2} / d t}+2 d L^{-1 / 2}\right\} \\
\leqq \frac{2 d}{(2 \pi t)^{d / 2}}\left\{\frac{\Gamma\left(1+\frac{1}{\alpha}\right)}{t^{1 / \alpha}} e^{-2 L / d t}+2 d L^{-1 / 2}\right\} \leqq \frac{2 d}{t^{r_{4}}} \sup _{t \in[0, \infty)} t^{r_{4}-d / 2-1 / \alpha} e^{-L / d t} \Gamma\left(1+\frac{1}{\alpha}\right)
\end{gathered}
$$




$$
+\frac{4 d^{2}}{t^{d / 2}} L^{-1 / 2} \leqq \frac{a_{5}}{t^{r_{4}}} L^{r^{4}-d / 2-1 / \alpha}+\frac{a_{6}}{t^{r_{6}}} L^{-1 / 2} t^{r_{6}-d / 2} .
$$

In order to satisfy the lemma we have the requirements $r_{4}>1, d / 2+1 / \alpha>r_{4}$ for the first term and $r_{6}>1, r_{6}-d / 2>0,\left(r_{6}-d / 2\right) \frac{2 \alpha}{\alpha+2}<\frac{1}{2}$ for the second term. These requirements can be satisfied for $d / 2+1 / \alpha>1$. Finally we have

$$
\begin{aligned}
& \frac{1}{(2 \pi t)^{d / 2}} \int_{0}^{1} d u_{1} e^{-t u_{1}^{\alpha}} \int_{0}^{1} d v_{1} \int_{0}^{t} d \tau e^{-t L^{2}\left|v_{1}-u_{1}\right|^{2} / 2 \tau(t-\tau)}\left(\frac{t L^{2}}{2 \pi \tau(t-\tau)}\right)^{1 / 2} \cdot\left|v_{1}^{\alpha}-u_{1}^{\alpha}\right| \\
& \leqq \frac{L}{(2 \pi t)^{d / 2}} \int_{0}^{t} d \tau \cdot\left(\frac{t}{2 \pi \tau(t-\tau)}\right)^{1 / 2} \int_{0}^{1} d u_{1} \cdot e^{-t u_{1}^{\alpha}} \int_{0}^{1} d \tau e^{-\inf _{\tau \in[0, t]}\left(t L^{2}\left|v_{1}-u_{1}\right|^{2} / 2 \tau(t-\tau)\right)} \cdot\left|v_{1}^{\alpha}-u_{1}^{\alpha}\right| \\
& \leqq \frac{L}{t^{(d-1) / 2}} \int_{0}^{1} d u_{1} e^{-t u_{1}^{\alpha}} \int_{0}^{1} d v_{1} \cdot\left|v_{1}^{\alpha}-u_{1}^{\alpha}\right| e^{-L^{2}\left|v_{1}-u_{1}\right|^{2} / t} .
\end{aligned}
$$

If we substitute $v_{1}=p u_{1}$ and if we use $\left|p^{\alpha}-1\right| \leqq 2^{\alpha}|p-1|^{\alpha}$ for $p \geqq 2$ and $|p-1|^{\alpha} \leqq 2^{\alpha}|p-1|$ for $0 \leqq p \leqq 2$ we have

$$
\begin{aligned}
\leqq & \frac{L \cdot 2^{\alpha}}{t^{(d-1) / 2}} \int_{0}^{1} d u_{1} e^{-t u_{1}^{\alpha}} \cdot u_{1}^{\alpha+1}\left\{\int_{0}^{2} d p \cdot|p-1| \cdot e^{-L^{2}|p-1|^{2} u_{1}^{2} / t}\right. \\
& \left.+\int_{2}^{\infty} d p \cdot|p-1|^{\alpha} \cdot e^{-L^{2} u_{1}^{2}|p-1|^{2} / t}\right\} \\
\leqq & \frac{L \cdot 2^{\alpha}}{t^{(d-1) / 2}} \int_{0}^{\infty} d u_{1} e^{-t u_{1}^{\alpha}} \cdot u_{1}^{\alpha+1}\left\{\int_{-\infty}^{+\infty} d p \cdot e^{-L^{2}|p-1|^{2} u_{1}^{2} / t}\left(|p-1|+|p-1|^{\alpha}\right)\right\} \\
\leqq & \frac{a_{7}}{L t^{(d-1) / 2}}+\frac{a_{8}}{L^{\alpha} t^{d / 2+1 / \alpha-\alpha / 2-1}} .
\end{aligned}
$$

In the same way as before we see that for $d / 2+1 / \alpha>1$ and $t<T(L)$ suitable constants $r_{8}, \ldots, r_{11}$ can be found.

Lemma 4. For $z \in[0,1]$

$$
\lim _{L \rightarrow \infty} f_{L}(z)=\frac{1}{(2 \pi)^{d / 2}} \int_{0}^{1} d u g_{d / 2}\left(z e^{-u^{\alpha}}\right) .
$$

Proof. We have the following estimate:

$$
\begin{aligned}
& \left|f_{L}(z)-\frac{1}{(2 \pi)^{d / 2}} \int_{0}^{1} d u g_{d / 2}\left(z e^{-u^{\alpha}}\right)\right|=\left|\sum_{n=1}^{\infty} z^{n} S_{L}^{\prime}(n)-\sum_{n=1}^{\infty} z^{n} G(n)\right| \\
& \leqq \\
& \sum_{n=1}^{\infty}\left|S_{L}^{\prime}(n)-G(n)\right| \leqq \sum_{n=1}^{T(L)}\left|S_{L}^{\prime}(n)-G(n)\right|+\sum_{n=T(L)+1}^{\infty} S_{L}^{\prime}(n)+\sum_{n=T(L)+1}^{\infty} G(n) \\
& \leqq \\
& \quad \sum_{n=1}^{T(L)}\left|S_{L}(n)-G(n)\right|+\sum_{n=1}^{T(L)}\left|S_{L}^{\prime}(n)-S_{L}(n)\right| \\
& \quad+\sup _{n \geqq T(L)+1} n^{r_{1}} S_{L}^{\prime}(n) \sum_{n=1}^{\infty} n^{-r_{1}}+\sum_{n=T(L)+1}^{\infty} G(n) .
\end{aligned}
$$


By Lemma 3 we have

$$
\begin{aligned}
\sum_{n=1}^{T(L)}\left|S_{L}(n)-G(n)\right| & \leqq \sum_{n=1}^{T(L)}\left(\frac{a_{4}}{n^{r_{2}} L^{r_{3}}}+\ldots+\frac{a_{8}}{n^{r_{10}} L^{r_{11}}}\right) \\
& \leqq \frac{a_{4}}{L^{r^{3}}} g_{r_{2}}(1)+\ldots \frac{a_{8}}{L^{r_{11}}} g_{r_{10}}(1)
\end{aligned}
$$

Furthermore for $L>1$

$$
\begin{aligned}
& \sum_{n=1}^{T(L)}\left|S_{L}^{\prime}(n)-S_{L}(n)\right|=\frac{1}{L^{d}} \sum_{n=1}^{T(L)} \sum_{\left\{k: k_{1}=1\right\}} e^{-n \eta_{k}^{L}} \leqq \frac{1}{L} \sum_{n=1}^{T(L)} \frac{e^{\left(r_{1} / 2 a_{2}\right)(d-1) \pi^{2}}}{n^{(d-1) / 2}} \\
& \quad \leqq\left\{\begin{array}{l}
\frac{T(L)}{L},(d=1) \\
\frac{e^{\left(r_{1} / 2 a_{2}\right)(d-1) \pi^{2}}}{L}\left(1+2(T(L))^{1 / 2}\right), \quad(d \geqq 2),
\end{array}\right.
\end{aligned}
$$

where we have used the fact that for $d=1, \eta_{1}=0$ and for $d \geqq 2$ the sum is the partition function for a $(d-1)$-dimensional cube with volume $L^{d-1}$. We have used the upperbound from Lemma 2. Because $T(L) \sim L^{2 \alpha /(\alpha+2)}, \frac{d}{2}+\frac{1}{\alpha}>1$, this term approaches zero as $L \rightarrow \infty$. Now we estimate sup $n^{r_{1}} S_{L}^{\prime}(n)$. Since $n^{r_{1}} e^{-n \eta}$ is monotonically decreasing as a function of $n$ for $n>r_{1} / \eta$, the sum $n^{r_{1}} S_{L}^{\prime}(n)$ is a decreasing function of $n$ for

$$
n>r_{1} \cdot\left(\inf _{\left\{k: k_{1} \neq 1\right\}} \eta_{k}^{L}\right)^{-1}=\frac{r_{1}}{\varepsilon_{2}^{L}-\varepsilon_{1}^{L}}
$$

Since $\frac{r_{1}}{\varepsilon_{2}^{L}-\varepsilon_{1}^{L}} \leqq T(L)+1$ for large $L$ we have

$$
\begin{aligned}
& \quad \sup _{n \geqq T(L)+1} n^{r_{1}} S_{L}^{\prime}(n)=(T(L)+1)^{r_{1}} S_{L}^{\prime}(T(L)+1) \leqq(T(L)+1)^{r_{1}} S_{L}^{\prime}(T(L)) \\
& \quad \leqq(T(L)+1)^{r_{1}} S_{L}(T(L)) \leqq(T(L)+1)^{r_{1}}\left\{\left|S_{L}(T(L))-G(T(L))\right|+G(T(L))\right\} .
\end{aligned}
$$

If we use Lemma 3 and $G(T(L)) \leqq(T(L))^{-d / 2-1 / \alpha} \Gamma(1+1 / \alpha)$ and choose $r_{1}=\inf \left\{r_{2}, r_{4}, \ldots, r_{10}, \frac{1}{2}+\frac{d}{4}+\frac{1}{2 \alpha}\right\}$, Lemma 4 follows since the fourth term in the estimate is trivial.

Lemma 4 enables us to prove Theorem 1 if $d / 2+1 / \alpha>1$. So we will consider only those cases in which $\rho_{c}<\infty$.

Proof of Theorem 1. We write the equation for $\zeta(L)$ in the following way

$$
f_{L}(\zeta(L))+\frac{1}{L^{d}} \sum_{n=1}^{\infty}(\zeta(L))^{n} \sum_{\left\{k: k_{1}=1\right\}}^{\infty} e^{-t \eta_{k}^{L}}=\rho .
$$

Consider first the case 1: $\rho<\rho_{c}$. Then there exists an $\varepsilon>0$ such that $\rho+\varepsilon<\rho_{c}$ and by Lemma 3 an $L_{0}(\varepsilon)$ such that for $L>L_{0}(\varepsilon)$ 


$$
\frac{1}{(2 \pi)^{d / 2}} \int_{0}^{1} d u g_{d / 2}\left(\zeta(L) e^{-u^{\alpha}}\right)<f_{L}(\zeta(L))+\varepsilon<\rho+\varepsilon<\rho_{c} .
$$

Let $\zeta_{\varepsilon}$ be the unique solution of

$$
\frac{1}{(2 \pi)^{d / 2}} \int_{0}^{1} d u g_{d / 2}\left(\zeta_{\varepsilon} e^{-u^{\alpha}}\right)=\rho+\varepsilon,
$$

then $\zeta(L)<\zeta_{\varepsilon}<1$. Let $\zeta$ be the unique solution of

$$
\rho=\frac{1}{(2 \pi)^{d / 2}} \int_{0}^{1} d u g_{d / 2}\left(\zeta e^{-u^{\alpha}}\right)
$$

then

$$
\begin{aligned}
& |\zeta(L)-\zeta| \leqq \frac{\frac{1}{(2 \pi)^{d / 2}} \int_{0}^{1} d u g_{d / 2}\left(\zeta(L) e^{-u^{\alpha}}\right)-\rho}{\frac{1}{(2 \pi)^{d / 2}} \inf _{\theta \in[0,1]} \frac{d}{d \theta} \int_{0}^{1} d u g_{d / 2}\left(\theta e^{-u^{\alpha}}\right)} \\
& \leqq(2 \pi)^{d / 2} \cdot e\left\{\left|f_{L}(\zeta(L))-\rho\right|+\left|f_{L}(\zeta(L))-\frac{1}{(2 \pi)^{d / 2}} \int_{0}^{1} d u g_{d / 2}\left(\zeta(L) e^{-u^{\alpha}}\right)\right|\right\} \\
& \leqq(2 \pi)^{d / 2} \cdot e\left\{\frac{1}{L^{d}} \sum_{n=1}^{\infty}(\zeta(L))^{n} \sum_{\left\{k: k_{1}=1\right\}} e^{-n \eta_{k}^{L}}+\varepsilon\right\} \\
& \leqq(2 \pi)^{d / 2} \cdot e\left\{\frac{2^{d-1}}{L} \frac{\zeta(L)}{1-\zeta(L)}+\varepsilon\right\} \\
& \leqq(2 \pi)^{d / 2} \cdot e\left\{\frac{2^{d-1}}{L} \frac{\zeta_{\varepsilon}}{1-\zeta_{\varepsilon}}+\varepsilon\right\} \text {, }
\end{aligned}
$$

where we have used (6.3) and Lemma 3.

Consider the case $\rho \geqq \rho_{c}$ : Given an $\varepsilon>0$ and choose a $\delta$ such that

$$
2 \delta<\rho_{c}-\frac{1}{(2 \pi)^{d / 2}} \int_{0}^{1} d u g_{d / 2}\left((1-\varepsilon) e^{-u^{\alpha}}\right),
$$

then there exists an $L_{0}$ such that for $L>L_{0}$

$$
\frac{1}{L^{d}} \sum_{n=1}^{\infty}(1-\varepsilon)^{n} \sum_{\left\{k: k_{1}=1\right\}} e^{-n \eta k}<\delta
$$

and by Lemma 3

$$
f_{L}(1-\varepsilon)<\frac{1}{(2 \pi)^{d / 2}} \int_{0}^{1} d u g_{d / 2}\left((1-\varepsilon) e^{-u^{\alpha}}\right)+\delta
$$

So

$$
\frac{1}{L^{d}} \sum_{n=1}^{\infty}(1-\varepsilon)^{n} \sum_{\left\{k: k_{1}=1\right\}} e^{-n \eta_{k}^{L}}+f_{L}(1-\varepsilon)<\frac{1}{(2 \pi)^{d / 2}} \int_{0}^{1} d u g_{d / 2}\left((1-\varepsilon)^{-u^{\alpha}}\right)+2 \delta
$$




$$
<\rho_{c} \leqq \rho=\frac{1}{L^{d}} \sum_{n=1}^{\infty}(\zeta(L))^{n} \sum_{\left\{k: k_{1}=1\right\}} e^{-n \eta_{k}^{L}}+f_{L}(\zeta(L))
$$

Moreover

$$
\frac{1}{L^{d}} \sum_{n=1}^{\infty} z^{n} \sum_{\left\{k: k_{1}=1\right\}} e^{-n \eta_{k}^{L}}+f_{L}(z)
$$

is monotonically increasing as a function of $z$, so $1-\varepsilon<\zeta(L)<1$ for all $L>L_{0}$ so $\zeta(L) \rightarrow 1$. Hence by Lemma 3 we have

$$
\frac{1}{L^{d}} \sum_{n=1}^{\infty}(\zeta(L))^{n} \sum_{\left\{k: k_{1}=1\right\}} e^{-n \eta_{k}^{L}} \rightarrow \rho-\rho_{c} .
$$

So in one dimension we have

$$
\frac{1}{L} \frac{\zeta(L)}{1-\zeta(L)} \rightarrow \rho-\rho_{c}, \quad(d=1) .
$$

In three or more dimensions we write

$$
\frac{1}{L^{d}} \frac{\zeta(L)}{1-\zeta(L)}+\frac{1}{L^{d}} \sum_{n=1}^{\infty}(\zeta(L))^{n} \sum_{\left\{k: k_{1}=1, k \neq(1, \ldots \ldots, 1)\right\}} e^{-n \eta_{k}^{L}} \rightarrow \rho-\rho_{c} .
$$

The terms, with one or more $k_{i}=1(i=2, \ldots, d)$ can easily be shown to vanish as $L \rightarrow \infty$. The sum of the remaining terms we estimate as follows. We obtain, using (2.17)

$$
\begin{aligned}
& \frac{1}{L^{d}} \sum_{n=1}^{\infty}(\zeta(L))^{n}\left(\sum_{k=2}^{\infty} e^{-\left(\pi^{2} n / 2 L^{2}\right)\left(k^{2}-1\right)}\right)^{2}\left(\sum_{k=2}^{\infty} e^{-\left(\pi^{2} n / 2 L^{2}\right)\left(k^{2}-1\right)}\right)^{d-3} \\
& \quad \leqq \frac{1}{L^{d}} \sum_{n=1}^{\infty} 2\left(e^{-\left(3 \pi^{2} n / L^{2}\right)}+\int_{2}^{\infty} \int_{2}^{\infty} e^{-\left(\pi^{2} n / 2 L^{2}\right)\left(k_{1}^{2}+k_{2}^{2}-2\right)} d k_{1} d k_{2}\right)\left(\sum_{k=1}^{\infty} e^{-\left(\pi^{2} n / 2 L^{2}\right) k^{2}}\right)^{d-3} \\
& \quad \leqq \frac{1}{L^{d}} \sum_{n=1}^{\infty} 2\left(e^{-\left(3 \pi^{2} n / L^{2}\right)}+\frac{L^{2}}{2 \pi n} e^{-\left(\pi^{2} n / L^{2}\right)}\right) L^{d-3} \\
& \quad \leqq \frac{2}{L^{3}} \frac{1}{e^{3 \pi^{2} / L^{2}}-1}-\frac{1}{\pi L} \log \left(1-e^{\left.-\pi^{2} / L^{2}\right)}\right.
\end{aligned}
$$

where we have used the first bound of Lemma 2 for a $(d-3)$-dimensional cube with volume $L^{d-3}$. So for $d \geqq 3$ as $L \rightarrow \infty$

and for $d=2$

$$
\frac{1}{L^{d}} \frac{\zeta(L)}{1-\zeta(L)} \rightarrow \rho-\rho_{c}
$$

$$
\frac{1}{L^{2}} \sum_{k=1}^{\infty} \frac{\zeta(L)}{e^{\left(k^{2}-1\right)\left(\pi^{2} / 2 L^{2}\right)}-\zeta(L)} \rightarrow \rho-\rho_{c}
$$

so

$$
\zeta(L) \sim 1-\frac{1}{A L^{2}},
$$


where $A$ is the unique solution of

$$
\sum_{k=1}^{\infty} \frac{1}{\left(k^{2}-1\right) \pi^{2} / 2+1 / A}=\rho-\rho_{c} .
$$

Proof of Theorem 2. We have

$$
\begin{aligned}
\mid p_{L} & -\frac{1}{(2 \pi)^{d / 2}} \int_{0}^{1} d u g_{(d+2) / 2}\left(\zeta e^{-u^{\alpha}}\right)|\leqq| p_{L}-\frac{1}{(2 \pi)^{d / 2}} \int_{0}^{1} d u g_{(d+2) / 2}\left(\zeta(L) e^{-u^{\alpha}}\right) \mid \\
& +\left|\int_{0}^{1} d u g_{(d+2) / 2}\left(\zeta e^{-u^{\alpha}}\right)-\int_{0}^{1} d u g_{(d+2) / 2}\left(\zeta(L) e^{-u^{\alpha}}\right)\right| \\
\leqq & f_{L}(\zeta(L))-\frac{1}{(2 \pi)^{d / 2}} \int_{0}^{1} d u g_{d / 2}\left(\zeta(L) e^{-u^{\alpha}}\right)|+| \int_{0}^{1} d u g_{(d+2) / 2}\left(\zeta e^{-u^{\alpha}}\right) \\
& -\int_{0}^{1} d u g_{(d+2) / 2}\left(\zeta(L) e^{-u^{\alpha}}\right)\left|+\sum_{n=1}^{\infty} \frac{(\zeta(L))^{n}}{n}\right| S_{L}(n)-S_{L}^{\prime}(n) \mid .
\end{aligned}
$$

The first term tends to zero as $L$ tends to infinity because of Lemma 3; the second term tends to zero because $\zeta(L) \rightarrow \zeta$ and $\int_{0}^{1} d u g_{(d+2) / 2}\left(z e^{-u^{\alpha}}\right)$ is a continuous, bounded function for $z \in[0,1]$. Finally the third term is majorized by $-\frac{2^{d-1}}{L} \log (1-\zeta(L))$. This tends to zero as $L$ tends to infinity.

Before proving Theorem 3 we give some definitions and two lemmas:

Definitions.

$$
\begin{aligned}
& Q_{L}(t, u)=\sum_{\{k\}} e^{-t \eta_{k}^{L}} \Psi_{k}^{2}(L u), \\
& Q_{L}^{\prime}(t, u)=\sum_{\left\{k: k_{1} \neq 1\right\}} e^{-t \eta_{k}^{L}} \Psi_{k}^{2}(L u), \\
& \Psi_{k}^{2}(L u)=\Phi_{k_{1}}^{2}\left(L u_{1}\right) \prod_{m=2}^{d}\left(\frac{2}{L}\right) \sin ^{2} \pi k_{m} u_{m}
\end{aligned}
$$

where $\Phi_{k_{1}}\left(L u_{1}\right)$ is defined in Sect. 2 .

Lemma 5. For all $u_{1}>u_{0}, \Phi_{1}^{2}\left(L u_{1}\right)$ is bounded by

$$
\Phi_{1}^{2}\left(L u_{1}\right) \leqq \frac{2}{3} \varepsilon_{1}^{L} \cdot L^{3 / 2} e^{-\int_{L u_{0}}^{L u_{1}}\left((x / L)^{\alpha}-\varepsilon_{1}^{L}\right)^{1 / 2} d x}
$$

where $u_{0}=\left(\varepsilon_{1}^{L}\right)^{1 / \alpha}$.

Proof. The proof can be extracted from [10].

Lemma 6. For all $d_{u, 1}>L^{-\delta}$ and $\varepsilon>0$ there exists an $L_{0}(\varepsilon)$ such that for $L>L_{0}(\varepsilon)$

$$
\left|\sum_{n=1}^{\infty}(\zeta(L))^{n} Q_{L}^{\prime}(n, u)-\sum_{n=1}^{\infty} \zeta^{n} \frac{e^{-n u_{1}^{\alpha}}}{(2 \pi n)^{d / 2}}\right|<\varepsilon .
$$


Proof of lemma 6. We have the following estimate:

$$
\begin{aligned}
& \left|\sum_{n=1}^{\infty}(\zeta(L))^{n} Q_{L}^{\prime}(n, u)-\sum_{n=1}^{\infty} \zeta^{n} \frac{e^{-n u_{1}^{\alpha}}}{(2 \pi n)^{d / 2}}\right| \\
& \leqq \sum_{n=1}^{T(L)}\left|Q_{L}(n, u)-\frac{e^{-n u_{1}^{\alpha}}}{(2 \pi n)^{d / 2}}\right|+\sum_{n=T(L)+1}^{\infty} \frac{e^{-n u_{1}^{\alpha}}}{(2 \pi n)^{d / 2}} \\
& +\sum_{n=T(L)+1}^{\infty} Q_{L}^{\prime}(n, u)+\sum_{n=1}^{\infty} \frac{\left|\zeta^{n}-(\zeta(L))^{n}\right|}{(2 \pi n)^{d / 2}} e^{-n u_{1}^{\alpha}} \\
& +\sum_{n=1}^{T(L)} \Phi_{1}^{2}\left(L u_{1}\right) \prod_{m=2}^{d} \sum_{k_{m}=1}^{\infty} e^{-\left(n \pi^{2} / 2 L^{2}\right)\left(k_{m}^{2}-1\right)} \cdot\left(\frac{2}{L}\right) \sin ^{2} \pi k_{m} u_{m} \equiv \mathrm{I}+\mathrm{II}+\mathrm{III}+\mathrm{IV}+\mathrm{V} .
\end{aligned}
$$

We estimate term by term

$$
\begin{aligned}
\mathrm{I} \leqq & \sum_{n=1}^{T(L)}\left|\sum_{k} e^{-n E_{k}^{L}}\left(\Psi_{k}(L u)\right)^{2}-\frac{e^{-n u_{1}^{\alpha}}}{(2 \pi n)^{d / 2}}\right|+\sum_{n=1}^{T(L)}\left(e^{n E_{1}^{L}}-1\right) \sum_{k} e^{-n E_{k}^{L}}\left(\Psi_{k}(L u)\right)^{2} \\
\leqq & \sum_{n=1}^{T(L)} \frac{e^{-n u_{1}^{\alpha}}}{(2 \pi n)^{d / 2}}\left\{2 d e^{-2 L^{2} d_{u, 2}^{2} / d n}\right. \\
& +\left|\int_{0}^{n} d \tau \int_{0}^{1} d x_{1}\left(u_{1}^{\alpha}-x_{1}^{\alpha}\right) \cdot\left(\frac{L^{2} n}{2 \pi \tau(n-\tau)}\right)^{1 / 2} e^{-n L^{2}\left|x_{1}-u_{1}\right|^{2} / 2 \tau(n-\tau)}\right| \\
& \left.+\left|\int_{0}^{n} \frac{d \tau}{n} \int_{0}^{1} d x_{1} \cdot\left(\frac{L^{2} n}{2 \pi \tau(n-\tau)}\right)^{1 / 2} e^{n\left(u_{1}^{\alpha}-x_{1}^{\alpha}\right)-n L^{2}\left|x_{1}-u_{1}\right|^{2} / 2 \tau(n-\tau)}-1\right|\right\} \\
& +\sum_{n=1}^{T(L)} e^{T(L) E_{1}^{L}} \cdot n E_{1}^{L} \frac{1}{(2 \pi n)^{d / 2}} \int_{0}^{n} \frac{d \tau}{n} \int_{0}^{1} d x_{1} e^{-n x_{1}^{\alpha}-n L^{2}\left|x_{1}-u_{1}\right|^{2} / 2 \tau(n-\tau)} \cdot\left(\frac{L^{2} n}{2 \pi \tau(n-\tau)}\right)^{1 / 2} \\
& \equiv \mathrm{VI}+\mathrm{VII}+\mathrm{VIII}+\mathrm{IX},
\end{aligned}
$$

where we have used (6.5) and (6.6). Furthermore we have enlarged the integration over the cube $B_{1}$ to a larger domain $D=\left\{x: 0 \leqq x_{1} \leqq 1,-\infty<x_{2}<\infty \ldots\right.$, $\left.-\infty<x_{d}<\infty\right\}$ and $d_{u, 2}=\inf \left\{u_{1}, 1-u_{1}\right\}$. We will not treat all the four terms but we will treat as an example the second one:

$$
\begin{aligned}
\mathrm{VII} & \leqq \sum_{n=1}^{T(L)} \frac{e^{-n u_{1}^{\alpha}}}{(2 \pi n)^{d / 2}} \int_{0}^{n} d \tau \int_{0}^{1} d x_{1}\left|u_{1}^{\alpha}-x_{1}^{\alpha}\right|\left(\frac{L^{2} n}{2 \pi \tau(n-\tau)}\right)^{1 / 2} \sup _{\tau \in[0, n]} e^{-n L^{2}\left|x_{1}-u_{1}\right|^{2} / 2 \tau(n-\tau)} \\
& \leqq \sum_{n=1}^{T(L)} \frac{e^{-n u_{1}^{\alpha}}}{(2 \pi n)^{d / 2}} \int_{0}^{n} d \tau \int_{0}^{1} d x_{1}\left|u_{1}^{\alpha}-x_{1}^{\alpha}\right|\left(\frac{L^{2} n}{2 \pi \tau(n-\tau)}\right)^{1 / 2} e^{-2 L^{2}\left|x_{1}-u_{1}\right|^{2} / n} \\
& \leqq \sum_{n=1}^{T(L)} \frac{e^{-n u_{1}^{\alpha}}}{n^{(d-1) / 2}} \cdot L \int_{0}^{1} d x_{1}\left|u_{1}^{\alpha}-x_{1}^{\alpha}\right| e^{-L^{2}\left|x_{1}-u_{1}\right|^{2} / n}
\end{aligned}
$$

We have estimated this integral before in (6.16). So

$\mathrm{VII} \leqq \sum_{n=1}^{T(L)} \frac{e^{-n u_{1}^{\alpha}}}{n^{(d-1) / 2}} L \cdot\left\{\frac{b_{1} n u_{1}^{\alpha-1}}{L^{2}}+\frac{b_{2} n^{(\alpha+1) / 2}}{L^{\alpha+1}}\right\}$ 


$$
\begin{aligned}
& \left.\leqq \sum_{n=1}^{T(L)} \frac{b_{1}}{n^{(d-1) / 2} L u_{1}} \sup _{n u_{1}^{\alpha} \in[0, \infty)} n u_{1}^{\alpha} e^{-n u_{1}^{\alpha}}+\frac{b_{1}}{n^{d / 2-\alpha / 2} L^{\alpha}} \sup _{n \in[0, \infty)} n e^{-n u_{1}^{\alpha}}\right\} \\
& \leqq \sum_{n=1}^{T(L)}\left\{\frac{b_{1}}{n^{(d-1) / 2} L u_{1}}+\frac{b_{2}}{n^{(d-\alpha) / 2} L^{\alpha} u_{1}^{\alpha}}\right\} \\
& =\sum_{n=1}^{T(L)}\left\{\frac{b_{1} n^{s_{1}}}{n^{(d-1) / 2+s_{1}} L u_{1}}+\frac{b_{2} n^{s_{2}+\alpha / 2}}{n^{d / 2+s_{2}}\left(L u_{1}\right)^{\alpha}}\right\} .
\end{aligned}
$$

We are able to find a constant $s_{1}$ such that $(d-1) / 2+s_{1}>1$ and $(T(L))^{s_{1}} \cdot\left(L u_{1}\right)^{-1} \rightarrow$ 0 for $u_{1}>L^{-\delta}$ with $\delta>0$ if $d / 2+1 / \alpha>1$. A similar argument holds for the second term.

We are able to treat all the other terms in a similar way. For instance, term III will be split up like in Lemma 4 and in term IV we use the convergence of $\zeta(L)$ to $\zeta$ for large $L$ (Theorem 1). In term V we have to use Lemma 5 to estimate $\Phi_{1}^{2}\left(L u_{1}\right)$. Proof of Theorem 3. We first consider $d=1$. In this case we have to show that

$$
\sum_{n=1}^{\infty}(\zeta(L))^{n} \Phi_{1}^{2}\left(L u_{1}\right) \rightarrow\left(\rho-\rho_{c}\right) \delta\left(u_{1}\right) .
$$

This is obvious since $\frac{1}{L} \frac{\zeta(L)}{1-\zeta(L)} \rightarrow \rho-\rho_{c}$ and $L \Psi_{1}^{2}\left(L u_{1}\right)$ is a normalized function of $u_{1}$ on $[0,1]$. Furthermore we have by Lemma 5 that $L \Phi_{1}^{2}\left(L u_{1}\right) \rightarrow 0$ for all $u_{1}>u_{0}$. Since $u_{0} \rightarrow 0$ for $L \rightarrow \infty, L \Phi_{1}^{2}\left(L u_{1}\right)$ tends to the $\delta$-distribution in the weak sense. Consider $d \geqq 3$. By a similar estimate as in the proof of Theorem 1 we show that

$$
\sum_{n=1}^{\infty}(\zeta(L))^{n} \prod_{m=2}^{d} \sum_{k_{m} \geqq 2}^{\infty} e^{-\left(\pi^{2} n / 2 L^{2}\right)\left(k_{m}^{2}-1\right)} \frac{2}{L} \sin ^{2} \pi k_{m} u_{m} \rightarrow 0
$$

for all $u_{2}, u_{3} \ldots u_{d}$. So the density distribution function of the condensate part is given by

$$
\left(\rho-\rho_{c}\right) \delta\left(u_{1}\right) \prod_{m=2}^{d} 2 \sin ^{2} \pi k_{m} u_{m} .
$$

Consider $d=2$. As in Theorem 1 it can be seen that all the terms $k_{2}=1,2, \ldots$ contribute to the one-particle distribution function of the condensate since the $\eta_{k}^{L}$ with $k_{1}=1$ scale with the volume. Thus with Theorem 1 we have

$$
\begin{gathered}
L \Phi_{1}^{2}\left(L u_{1}\right) \sum_{k=1}^{\infty} \frac{\zeta(L)}{e^{\left(k^{2}-1\right)\left(\pi^{2} / 2 L^{2}\right)}-\zeta(L)} \frac{2}{L^{2}} \sin ^{2} \pi k u_{2} \rightarrow \\
\delta\left(u_{1}\right) \cdot \sum_{k=1}^{\infty} \frac{2 \sin ^{2} \pi k u_{2}}{\left(k^{2}-1\right) \frac{\pi^{2}}{2}+\frac{1}{A}} .
\end{gathered}
$$

Acknowledgements. The first author wishes to thank N. M. Hugenholtz and J. V. Pulè for their helpful comments. This paper is part of the research program of the "Foundation for Fundamental Research on Matter", which is financially supported by the "Netherlands Organization for Pure Research". 


\section{References}

1. Van den Berg, M. : Phys. Lett. 78A, 88-90 (1980)

2. Ziff, R. M., Uhlenbeck, G. M., Kac, M. : Phys. Lett. 32C, 171-248 (1977)

3. Davies, E. B. : Commun. Math. Phys. 30, 229-247 (1973)

4. Landau, L. J., Wilde, I. F.: Commun. Math. Phys. 70, 43-51 (1979)

5. Lewis, J. T., Pulè, J. V. : Commun. Math. Phys. 36, 1-18 (1974)

6. Cannon, J. T. : Commun. Math. Phys. 29, 89-104 (1973)

7. Lewis, J. T., Pulè, J. V. : Commun. Math. Phys. 45, 115-131 (1975)

8. Critchley, R. H., Lewis, J. T. : Commun. Math. Phys. 44, 107-124 (1975)

9. Van den Berg, M. : Bounds on Green's functions of second-order differential equations, preprint (1980)

10. Titchmarsh, E. C. : Eigenfunction Expansions I, Chap. VIII. Oxford: Clarendon Press 1962

11. Kac, M.: Proc. Second Berkeley Symposium on Mathematical Statistics and Probability, pp. 189-215 Berkeley: University of California Press 1951

Communicated by E. Lieb

Received January 19, 1981; in revised form February 17, 1981 